\title{
Study on Creep Property and Creep Model for the Rock Foundation of High Fill Carbonate
}

\begin{abstract}
Junyi Fu, Bin Wu, Hong Wang
Department of Guizhou Karst foundation engineering technology research center, Zhengye Engineering and Investment Incorporation Limited, Guiyang, 550000, China

Abstract-The carbonate foundation creep has significant difference between natural and moist condition under stress. Through field test of high fill carbonate foundation, it has shown that rocks have been in a long-term moist state, the total settlement of rocks creep in the moist state are significantly greater than the state of nature. In this paper, according to the difference between moist state and nature state of rocks creep, establishing the mechanical model of experience and creep in mechanical model, providing reference for the construction of KARST areas.
\end{abstract}

Keywords-creep, empirical, model creep model

\section{INTRODUCTION}

KARST areas are widely distributed especially in the southwest provinces of China. With the rapid development of infrastructure construction in China's southwest mountainous area, high fill foundation is increasing, it has direct influence on the normal use of facilities such as airports, highways, and railways [1]. At present, it has a lot of achievements on the study of the carbonate laboratory test, but for study on field testis are still in its infancy. Through the study of the observations on high fill carbonate rock stone foundation field test, this paper carried on the thorough research to the post-construction settlement of carbonate high fill foundation by means of regression parameters of the model and generalized Kelvin model.

\section{FOUNDATION STRATA DISTRIBUTION}

The experiment based on "coal(coke、 chemical)- SteelElectric" integration circular economy project. Composition of fill area from top to bottom: Quaternary strata (Q4pd) Soil, taupe, contain roots and residues, surface crack developed soon, distributing widely, thickness $0.3 \sim 0.8 \mathrm{~m}$; $(\mathrm{Q} 4 \mathrm{el}+\mathrm{dl})$ red clay: yellow-red, hard plastic-plastic, distributing widely, thickness $3 \sim 5 \mathrm{~m}$; Xiao Tuan Lao Bao underlying bedrock in the fill area is most of the thin layers of limestone, and a small amount of medium thick layer of argillaceous limestone.
The project of filler is natural stone in the excavation zone, the parent rock is the

triassic guanling group rock stratum, the rock is broken and mainly medium weathering. Rocks are mainly medium thick layer $\sim$ thick layer of dolomite、 thin layer $\sim$ thick layer of limestone and medium thick layer $\sim$ thick layer of argillaceous limestone. The content of filler clay is not more than $5 \%$, the filler all used natural gradation stones which particle size $\leq 500 \mathrm{~mm}$. Foundationtreatment method was dynamic compaction.

\section{CREEP EXPERIMENT FACILITY}

The experimental platforms arranged the test point in sequence, the plane layout of every experimental

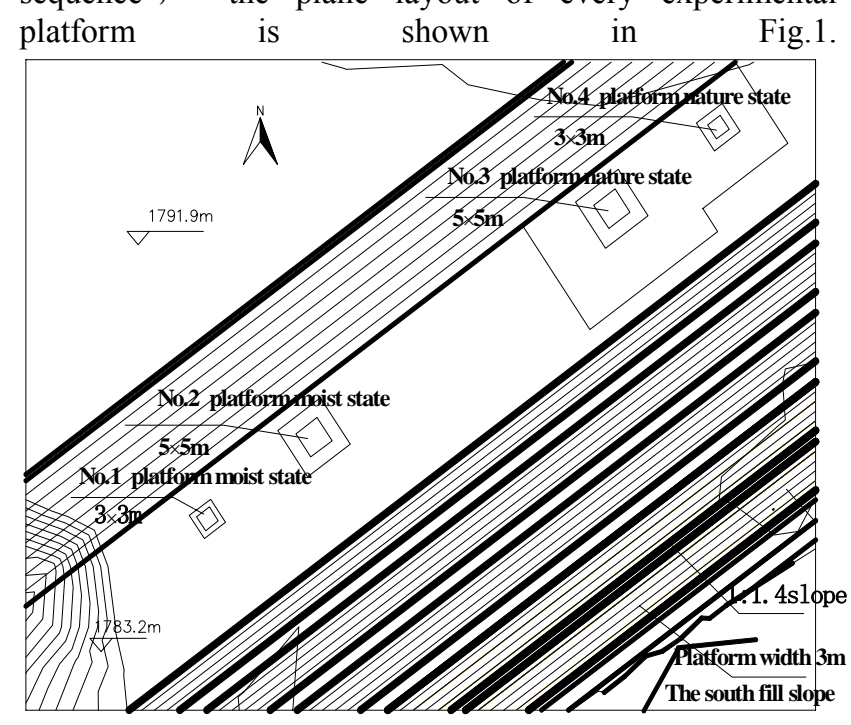

Figure 1. Experimental platform plan 


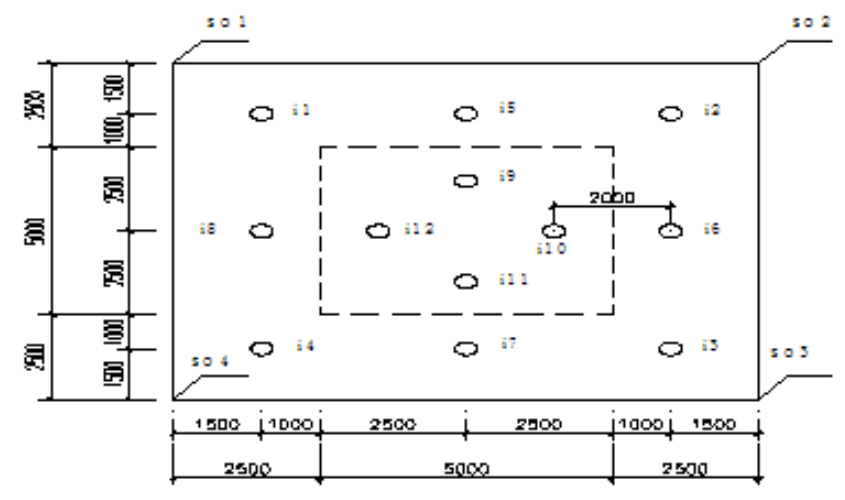

Figure 2. 5.0 $\times 5.0 \mathrm{~m}$ experimental platform

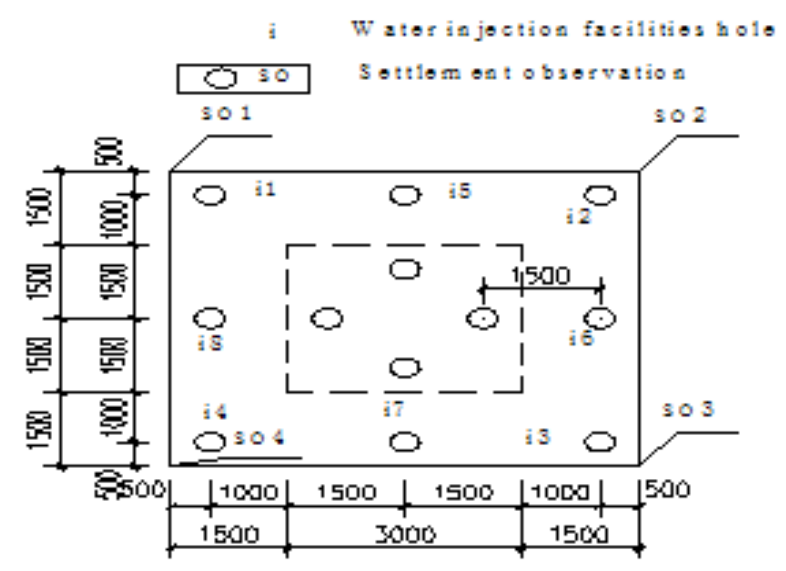

Figure 3. 3.0 $\times 3.0 \mathrm{~m}$ experimental platform

Among them the bottom size of No. 1 and No. 4 experimental platform was $3.0 \mathrm{~m} \times 3.0 \mathrm{~m}$, the bottom size of No. 2 and No. 3 experimental platform was $5.0 \mathrm{~m} \times 5.0 \mathrm{~m}$, the creep experiment in the moist state was carried out through the No. 1 and No. 2 experimental platforms, the creep experiment in the nature state was carried out through the No. 3 and No. 4 experimental platforms. The platform observation and water injection device are shown in Fig.2 and Fig.3.

\section{EXPERIMENTAL RESULTS}

The way 4 experimental platforms loading mode was eight steps loading, loading lasted 101 days, keeping the bottom stress of all experimental platforms were $420 \mathrm{KPa}$ after the completion of the final loading. The experiment of No. 1 and No. 2 experimental platforms were carried out in the moist state, the depth of moist gravel packing and creep influence were 5 times width of $5 \times 5 \mathrm{~m}$ experimental platforms and 4.5 times width of $3 \times 3 \mathrm{~m}$ experimental platforms, that is, $25 \mathrm{~m}$ and $13.5 \mathrm{~m}$, the angle of water diffusion was $30^{\circ}$.Keeping settlement observation to the 315 th day, curve of the relationship between the experimental platforms of settlement and time had been obtained, as is shown in Fig.4.

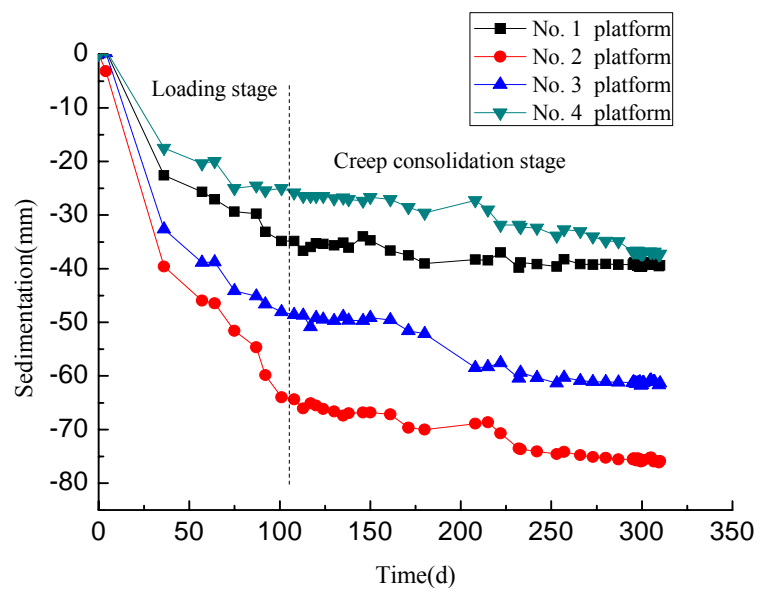

Figure 4. Experimental platform settlement curve

After creep experiment, through the comparisons of the same size of the experimental platforms we can draw that the total settlement of No.1 platform in moist state significantly greater than the total settlement of No.4 platform in nature state, the total settlement of No.2 platform in moist state significantly greater than the total settlement of No.3 platform in nature state, it can be obtained that the foundation creep has been affected by moist state. Through the comparisons of the same state of the No.1 and No.2 platforms we can draw that the total settlement of No.2 platform significantly greater than the total settlement of No.1 platform, it can be thought that the size of the experimental platforms and platform sedimentation value are a positive relationship, this phenomenon compared to No.3 and No.4 platforms which in nature state can also be obtained. The maximum subsidence value of four platforms under the stress was No.2 subsidence value $75.54 \mathrm{~mm}$, the experiments show that no matter in the nature or moist state, rock foundation of high fill carbonate can be meet the specification requirements for the foundation deformation settlement of 《Code for design of building foundation》 [2].

\section{CREEP MODEL}

\section{A Empirical mode}

The sedimentation process of the experiment is divided into two stages: instantaneous settlement of loading stage and creep settlement after loading stage. Empirical formula using hyperbolic function as shown below[3]:

$$
\varepsilon(\mathrm{t})=-\mathrm{t} /(\mathrm{A}+\mathrm{Bt})
$$

Type $\mathrm{A}, \mathrm{B}$ are the regression constants, $\mathrm{t}$ is the duration of creep. Using regression analysis, the parameters of settlement relationship over time of all platforms can be obtained as shown in Table 1. 
TABLE 1. REGRESSION PARAMETERS OF THE MODEL

\begin{tabular}{ccccc}
\hline $\begin{array}{c}\text { Experiment } \\
\text { platform }\end{array}$ & $\begin{array}{c}\text { State of the } \\
\text { platform }\end{array}$ & A & B & R \\
\hline 1 & Moist & 0.85 & 0.022 & 0.98 \\
2 & Moist & 0.53 & 0.011 & 0.99 \\
3 & Nature & 0.74 & 0.014 & 0.98 \\
4 & Nature & 1.46 & 0.025 & 0.96 \\
\hline
\end{tabular}

Formula (1) fitting curve is shown in Fig.5, degree of fitting is very good. There are lots of creep experience formula, the form of formulas are also different because of rock composition structure stress and temperature, pay attention to the condition when using[4].

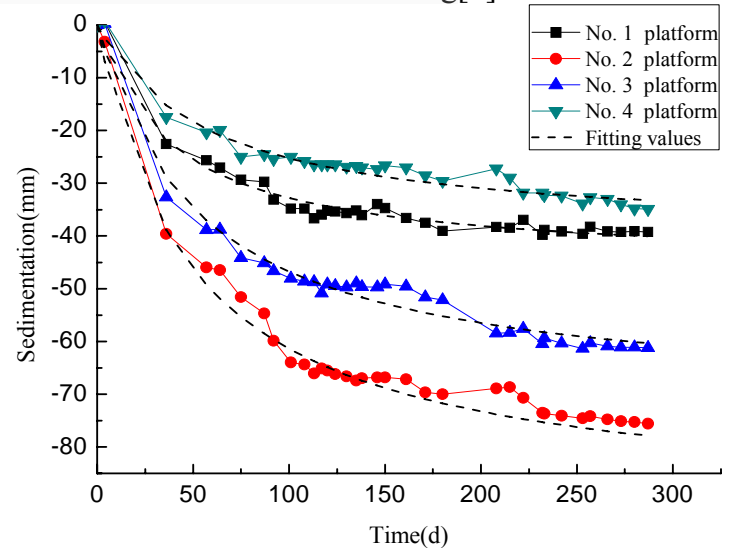

Figure 5. Experimental platforms settlement curve、 fitting curve

\section{B Generalized Kelvin model}

The whole process of carbonate rock stress and axial strain includes four steps ${ }^{[5]}$ : compaction、 elastic、strainhardening、 strain softening. In the practical engineering, the rock foundation is always in the second stage of stage, which carbonate rock creep stage contains the instantaneous deformation and creep viscosity stage. The creep value tends to a constant when time tends to infinity, which looks similar to the Kelvin model $[6,7,8]$. So, elastic element and Kelvin model should be included in creep model, we choose the Generalized Kelvin model for creep model here. There is no theoretical model which can describe a creep characteristics of residual deformation for now, so the Generalized Kelvin model is only applicable to the loaded state and is not applicable to unloading state [9]. The creep equation can be shown as follow.

$$
\varepsilon=\frac{\sigma_{0}}{k_{1}}+\frac{\sigma_{0}}{k_{2}}\left(1-e^{-\frac{k_{2}}{\eta} t}\right)
$$

Where $\sigma_{0}$ is a constant stress, $k_{1} 、 k_{2}$ are modulus of elasticity, $\eta$ is viscous coefficient.

Direct calculation method for parameters of $k_{1}$ 、 $k_{2}$ and $\eta$ is shown as follows:

$$
\begin{gathered}
k_{1}=\frac{\sigma_{1}}{\varepsilon_{1}} \\
k_{2}=\frac{\sigma_{1}}{\varepsilon(\infty)-\varepsilon_{1}}
\end{gathered}
$$

$\sigma_{1}$ is the loading stress, $\varepsilon_{1}$ is instantaneous strain, $\varepsilon(\infty)$ is strain when $t \rightarrow \infty$, Choosing any time $t>0$, it can be obtained by formula (2)

$$
\begin{gathered}
\varepsilon-\varepsilon_{1}=\frac{\sigma_{1}}{k_{2}}\left(1-e^{-\frac{k_{2}}{\eta} t}\right) \\
\eta=\frac{k_{2} t}{\ln \sigma_{1}-\ln \left[\sigma_{1}-k_{2}\left(\varepsilon-\varepsilon_{1}\right)\right]}
\end{gathered}
$$

Where, $\varepsilon$ is strain at any time.

The $\sigma_{0}$ of creep model mentioned above is constant stress, the field loading mode was eight steps in fact, the loading process is mainly instantaneous settlement, the settlement has been completed mainly in this process, settlement influence depth is that No.1 and No.4 experimental platforms are considered to be $13.5 \mathrm{~m}$; No.2 and No.3 experimental platforms are considered to be $25 \mathrm{~m}$. The loading phase of settlement is considered as instantaneous deformation of platforms under the $420 \mathrm{kPa}$ stress after completion of loading [10]. The creep parameters of No.2 and No.3 experimental platforms can be calculated directly, then the calculated values and experimental creep deformation values were compared in Fig.6, starting time which was set to 0 day was the 101th day after completion of loading. Then putting the creep model parameters of No.2 and No.3 experimental platforms as the creep model parameters of No.1 and No.4 platforms, then the creep deformation values of No.1 and No.4 platforms can be calculated and compared with the experimental values as shown in Fig.7. The calculation results were close to the experimental results. 


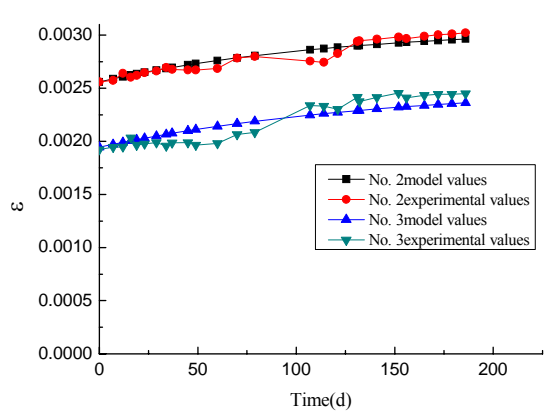

Figure 6. Platforms experiment, model curve

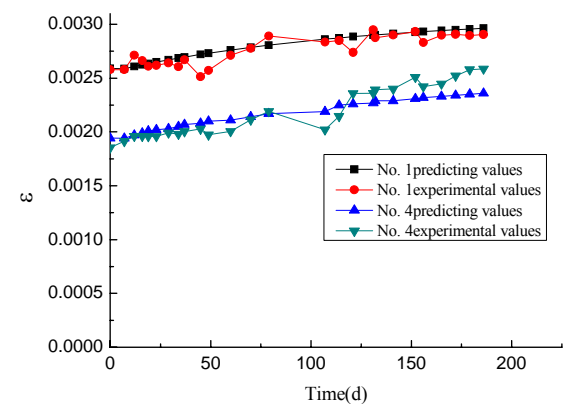

Figure 7. Platforms experiment, predicting curve

TABLE 2. CREEP MODEL PARAMETER VALUES OF NO.2 AND NO.3 PLATFORM

\begin{tabular}{ccccc}
\hline Platform & State & $k_{1}$ & $k_{2}$ & $\eta$ \\
\hline 2 & Moist & 164.06 & 821.92 & 98315.53 \\
3 & Nature & 216.05 & 735.55 & 103453.12 \\
\hline VI CONCLUSIONS & & & & VIII REFERENCES
\end{tabular}

Field experiments showed that, after completion of loading, the deformation of foundation settlement mainly occurred in the loading stage, the foundation creep rate significantly was slow down. The settlement of experiment platforms had tended to be stable at the end of the experiment, No.4 experimental platform sedimentation value was the smallest and No.2 experimental platform sedimentation value was the greatest, all the settlement values can satisfy the requirements of specification.

The experiments showed that, different size of the experimental platforms under the same condition, the larger platform bottom size was, the bigger creep settlement value was, but the deformation was consistent, this is mainly associated with the value of creep influence depth.

Using hyperbolic function fit out the empirical model of carbonate foundation through the creep test curve. Generalized kelvin model had been found as the creep model, the correlation between creep model curve and test curve was very good.

The settlement influence depth of experimental platforms is based on the basis of the experience, further experiments are needed to prove whether has error with real situation

\section{ACKNOWLEDGEMENTS}

The research work was supported by Science and Technology Department of Guizhou provincial under Grant No. Qian Ke He Ji Zi[2011]4002.
[1] Xu Ming, Song Er-Xiang. Review of long-term settling of high fills. Rock and soil mechanics, 49(6),pp.770-773, 2009.

[2] Industry standard of PRC. Code for design of building foundation(GB5007-2011). Beijing: China Building Industry Press, 2011.

[3] Liu Hong, Li Pan-Feng. Prediction of the post-construction settlement of high embankment of Jiuzhai-Huanglong airport. Journal of geotechnical engineering, 27(1), pp.90-93,2005.

[4] Jiang Yong-Dong, Xian Xue-Fu. Study on creep behaviors of sandstone and its mechanical models. Journal of geotechnical engineering, 27(12),pp.1478-1481, 2005.

[5] Cai Mei-Feng, He Man-Chao. Rock mechanics and engineering, Beijing: Science Press,2002.

[6] Okubo S. Fukui K., Long-term creep test and constitutive equation of Tage tuff . Journal of Mining and Materials, 118(1), pp.36-42, 2002.

[7] Chen Qu, Xi-Tian He-Fan. Creep behavior of Sedimentary soft rock under triaxial compression.Journal of rock mechanics and engineering, 22(6),pp.905-912,2003.

[8] Shen Zhen-Zhong, Xu Zhi-Ying. Creep test of granite for the Three Gorges Dam Foundation. Journal of Hohai University, 25(2),pp.17,1997 .

[9] Xiong Shi-Hu, Zhou Huo-Ming. Study of methodology of plate-loading creep test of rock mass. Journal of rock mechanics and engineering, 28(10), pp.2121-2127,2009.

[10] Song Er-Xiang, Cao Guang-Yu. Characteristics and simplified calculation method of creep settlement of high fill foundation in mountain area. Rock and soil mechanics, 33(6),pp.1711-1723, 2012. 